\title{
Ser homem é...: Adaptação da Escala de Concepções da Masculinidade
}

\author{
Valeschka Martins Guerra - Universidade Federal do Espirito Santo, Vitória, Brasil \\ Arielle Sagrillo Scarpati - Universidade Federal do Espírito Santo, Vitória, Brasil \\ Camila Nogueira Bonfim Duarte - Universidade Federal do Espirito Santo, Vitória, Brasil \\ Cleidiane Vitória da Silva - Universidade Federal do Espirito Santo, Vitória, Brasil \\ Tammy Andrade Motta - Universidade Federal do Espirito Santo, Vitória, Brasil
}

\begin{abstract}
Resumo
Esta pesquisa buscou adaptar a Escala de Concepções da Masculinidade (ECM) para o contexto brasileiro. O Estudo 1 investigou a estrutura fatorial da ECM, contando com a participação de 260 universitários, 56,8\% mulheres (Média de idade=22,2; $\mathrm{DP}=5,03)$. Os resultados sugeriram a existência de três dos quatro fatores esperados, além de uma redução na quantidade de itens, não corroborando a estrutura original. $\mathrm{O}$ Estudo 2 buscou comprovar a estrutura de três fatores e verificar a relação da masculinidade com a desejabilidade social. Participaram 220 usuários de internet, 53,4\% do sexo feminino (Média de idade=27; $\mathrm{DP}=8,35)$. Os resultados confirmaram a estrutura de três fatores proposta no Estudo 1 e apresentaram consistência interna aceitável. Foi observada uma correlação negativa da desejabilidade com o fator provocação social. Conclui-se que a ECM-16 possui características psicométricas satisfatórias e pode contribuir para uma melhor compreensão do fenômeno masculinidade. Palavras-chave: Masculinidade, Escala, Adaptação, Desejabilidade social.
\end{abstract}

To be a man is...: adapting the Meanings of Adolescent Masculinity Scale

\begin{abstract}
The main objective of this paper was to adapt the Meanings of Adolescent Masculinity Scale (MAMS) to the Brazilian context. Study 1 investigated the factor structure of the MAMS. There were 260 university students taking part in this study, 56.8\% women (Mean age=22.2; $\mathrm{SD}=5.03$ ). Results suggested the existence of only three of the four proposed factors, and also a reduction of the number of the items, which did not corroborate with the original structure. Study 2 aimed at confirming the three-factor structure and testing their relationship with social desirability. There were 220 internet users taking part in this study, 53.4\% women (Mean age=27; $\mathrm{SD}=8.35$ ). Results confirmed the three-factor structure proposed in Study 1 and presented acceptable internal consistency. The factor social teasing presented a negative correlation with social desirability. We have concluded that the scale presents adequate psychometric characteristics and could contribute with a better understanding of masculinity.

Keywords: Masculinity, Scale, Adaptation, Social desirability.
\end{abstract}

\section{Ser hombre es...: adaptación de la Escala de las Concepciones de Masculinidad}

\begin{abstract}
Resumen
Esta investigación buscó adaptar la Escala de las Concepciones de la Masculinidad (ECM) para el contexto brasileño. El estudio 1 investigó la estructura factorial de la ECM, con la participación de 260 universitarios, 56,8\% mujeres (edad media $=22,2$, $\mathrm{SD}=5,03)$. Los resultados indicaron la existencia de tres de los cuatro factores esperados, además de una reducción en la cantidad de ítems, lo que no corrobora la estructura original. El estudio 2 buscó comprobar la estructura de tres factores y la relación de masculinidad con la deseabilidad social. Participaron 220 usuarios de Internet, 53,4\% eran mujeres (edad media=27, $\mathrm{SD}=8,35)$. Los resultados confirmaron la estructura propuesta de los tres factores en el estudio 1 y presentaron consistencia interna aceptable. Hubo una correlación negativa de la deseabilidad con el factor provocación social. Se concluye que el ECM16 tiene características psicométricas satisfactorias y puede contribuir a una mejor comprensión del fenómeno masculinidad. Palabras clave: Masculinidad, Escala, Adaptación, Deseabilidad social.
\end{abstract}

\section{Introdução}

"The tragedy of machismo is that a man is never quite man enough" "Tradução livre: "A tragédia do machismo é que um homem nunca é homem suficiente"] (Germaine Gree, s/d, citada por Peter, 2010, p. 423).

As diferentes percepções existentes na sociedade atual do que é ‘ser homem' enfatizam a importância de se discutir a masculinidade a partir de uma perspectiva psicológica. O interesse pelo estudo deste tema começou a ser mais difundido a partir da década de 1960, gerando o surgimento de uma nova área da psicologia denominada 'a psicologia dos homens e da masculinidade' (Cochran, 2010). Nessa área, três paradigmas centrais foram propostos, resumindo as principais pesquisas e abordagens teóricas da época: 1) o paradigma biológico e evolucionista, que enfatiza os processos biológicos e evolutivos que influenciam no comportamento social tanto masculino como feminino; 2) o paradigma da identidade de 
gênero, que percebe esta identidade como um resultante natural do processo de desenvolvimento; e 3) o paradigma da tensão do papel de gênero, que propõe a construção cultural da masculinidade e a permanente tensão gerada pela tentativa, por parte dos homens, de agir de acordo com esse papel socialmente estabelecido (Cochran, 2010).

Tais paradigmas demonstram as diversas tentativas existentes na literatura psicológica de se compreender a masculinidade. Utilizado pela psicologia desde o início do século, o conceito de masculinidade pode ser entendido como um conjunto de características e habilidades que, significadas culturalmente, traduzem um estereótipo de homem. De acordo com Bonomo, Barbosa e Trindade (2008, p. 3), a masculinidade pode ser definida como "o sentimento de pertença em relação à categoria masculina". Connell (1997, p. 39), por sua vez, define masculinidade como "uma configuração de práticas em torno da posição dos homens na estrutura das relações de gênero". Esse autor ressalta, ainda, que existe uma pluralidade de configurações desse tipo em uma sociedade, devendo-se, portanto, falar de "masculinidades".

Não é possível, no entanto, falar desse conceito sem que antes se compreenda a construção da identidade de gênero, bem como das dinâmicas das relações entre homens e mulheres, a normatização da heterossexualidade e a categorização de grupo. Como afirma Grossi (2004, p. 5), este construto precisa ser considerado enquanto "uma categoria de análise". Ou seja, o conceito de gênero permite a problematização do que vem a ser o masculino e o feminino em nossa sociedade, uma vez que é por meio das experiências de gênero que homens e mulheres dão forma e significado às suas representações e práticas.

Sobre a identidade de gênero, Louro (1997) afirma que esta se trata da construção social referente ao ser homem e ser mulher, assim como a forma pela qual as pessoas se relacionam com o mundo, que tem origem nos espaços sociais. A construção de um gênero é interdependente da construção do outro, ou seja, para que um homem aprenda o que é ser homem, é necessário que ele tenha clareza do que significa ser mulher. Apenas assim ele poderá se manter afastado de qualquer prerrogativa social que o aproxime do universo feminino. Desse modo, não se acredita na existência de uma determinação natural dos comportamentos, mas sim, em uma construção social, histórica e cultural que pode ser organizada através do construto "gênero".

Normas sociais de gênero, advindas desse processo, influenciam, então, o comportamento de homens e mulheres e regulam as relações interpessoais, o que, atrelado a uma cultura patriarcal, favorece o surgimento da desigualdade. A normatização da heterossexualidade consequente dessa desigualdade resulta na produção do estereótipo, cuja função principal é facilitar a compreensão do outro no processo de socialização, tendo como base a categorização de grupo (Scardua \& Souza Filho, 2006).

As identidades são assumidas a partir do conflito estabelecido entre os grupos a que sentimos pertencer e os grupos a que não pertencemos (Tajfel, 1982). Para isso, todas as concepções que foram construídas historicamente sobre o ser homem nos mais diversos contextos (e.g., nas esferas familiar, escolar, médica, sexual) são contrastadas com as experiências reais do indivíduo, fazendo com que surjam ambiguidades expressas nas práticas e normas observadas no universo masculino na atualidade (Bonomo e cols., 2008). A não 'adequação' a essas normas implica, por sua vez, estereótipos negativos - tais como 'doente', 'anormal', 'antinatural' - que têm como objetivo o rebaixamento daqueles que não se encaixam nos papéis de gênero tradicionais. Como afirma Monteiro (1997), a hegemonia da masculinidade é construída mediante práticas sociais que enfatizam e reforçam normas e valores específicos.

Mediante um processo de aprendizagem e interiorização dessas normas e valores, presentes na educação de crianças e jovens, esses indivíduos constroem suas concepções do que é ser homem e ser mulher na sociedade ocidental. Estudos envolvendo a temática ganharam relevância nas últimas décadas no Brasil, em razão da percepção de que o referencial de masculinidade pode contribuir para dar maior visibilidade aos processos de saúde, risco e adoecimento da população masculina, assim como para fornecer subsídios para compreensão de relações violentas entre pares ou em relacionamentos românticos, por exemplo (Courtenay, 2000). Estes trabalhos chamaram atenção para o fato de que algumas práticas e comportamentos são adotados por homens no sentido de manter - a altos custos padrões hegemônicos de gênero, negando a existência da dor, do sofrimento e/ou da própria vulnerabilidade e reforçando um ideal de força masculina que demarca sua diferenciação com o feminino. Desse modo, as concepções de masculinidade influenciam na estruturação da identidade dos sujeitos (modelando seus comportamentos e emoções), assumindo a prerrogativa de modelos a serem seguidos (Oransky \& Fisher, 2009).

\section{Medindo aspectos da masculinidade}

Com o objetivo de compreender melhor a maneira como homens e mulheres entendem esse 
construto, surgem, ainda na década de 30, os primeiros instrumentos psicométricos que abordam a questão da masculinidade. A partir de então, vários instrumentos foram elaborados com o objetivo de medir diferentes aspectos do gênero.

Blazina, Pisecco e O’Neil (2005), por exemplo, propuseram a Gender Role Conflict Scale, com objetivo de avaliar níveis de conflito e estresse relacionados aos papéis de gênero em adolescentes. Um outro instrumento que poderia ser citado é o Bem Sex Role Inventory (BSRI), proposto por Bem (1974). Este é composto por escalas para as medidas de masculinidade e feminilidade, permitindo a classificação dos indivíduos em diferentes tipos de gênero em função de algumas das características de personalidade vinculadas aos papéis sexuais que o mesmo apresenta (Hernandez, 2009). Ressalta-se que o Inventário de Bem (1974) é, ainda hoje, um dos instrumentos mais conhecidos para avaliar qualquer medida relacionada a gênero, tendo sido adaptado ao contexto brasileiro (Hernandez, 2009). No entanto, tal instrumento tem como objetivo avaliar o quanto seus respondentes aderem a papéis sexuais masculino, feminino e/ou neutro, não questionando os participantes com relação à sua concordância ou não diante das normas de gênero propostas socialmente (Hernandez, 2009).

Para Mahalik e cols., (2003), a conformidade a normas masculinas é um construto multidimensional, que enfatiza a necessidade de aderir às expectativas sociais em face do seu papel de gênero. Com base nessas críticas, Oransky e Fisher (2009) desenvolveram um instrumento a fim de avaliar a rejeição ou concordância a normas masculinas de gênero por parte de adolescentes, uma vez que não existiam escalas multidimensionais que o fizessem satisfatoriamente.

\section{Escala de Concepsõos da Masculinidade (ECM)}

Para Oransky e Fisher (2009), a escala proposta por Blazina e cols. (2005), embora satisfatória em termos teóricos e estatísticos, se propõe a medir conflito e estresse relacionados aos papéis de gênero, enquanto a proposta da ECM foca na conformidade às normas, construto ainda não estudado de forma quantitativa no Brasil.

Para construir tal escala, esses autores criaram 60 itens provenientes de entrevistas abertas com adolescentes. Em seguida, três grupos focais foram realizados com o objetivo de selecionar os melhores itens e categorizá-los de acordo com quatro dimensões teóricas existentes na literatura sobre o tema. Os 34 itens restantes foram respondidos por 193 adolescentes do sexo masculino, recrutados em escolas dos Estados Unidos. As análises exploratórias realizadas eliminaram mais oito itens, sendo a versão final do instrumento composta por 26 itens, organizada nas quatro dimensões propostas teoricamente.

O primeiro fator, intitulado esforço constante, afirma que é necessário aparentar força e confiança constantemente para demonstrar masculinidade. De acordo com Vandello, Bossom, Cohen, Burnaford e Weaver (2008), a masculinidade é percebida como um estado precário, que necessita de constante confirmação para ser mantida.

O segundo fator, restrição emocional, afirma que para o indivíduo ser considerado masculino, deve esconder suas emoções e não aparentar vulnerabilidade. Essa dimensão é uma das mais estudadas das normas de gênero e tem sido consistentemente associada com a dificuldade que os homens apresentam de pedir ajuda (Tsan, Day, Schwartz, \& Kimbrel, 2011) e seus níveis de ansiedade (Wong, Pituch \& Rochlen, 2006).

O terceiro fator, heterossexismo, sugere que qualquer tipo de comportamento, por parte dos homens, que possa ser considerado feminino não é aceitável. O discurso de normatização da heterossexualidade é outra dimensão muito comum na literatura (Scardua \& Souza Filho, 2006). Murta, Del Prette e Del Prette (2010) sugerem que treinamentos direcionados para habilidades sociais poderiam contribuir para a diminuição de comportamentos de violência baseados na heteronormatividade.

Finalmente, o fator denominado provocação social inclui itens que sugerem que brincadeiras e piadas que jovens rapazes fazem uns com os outros devem ser aceitos como parte do 'ser homem'. Esta é a dimensão menos estudada na psicologia, tendo sido analisada apenas em pesquisas da linguística (Lampert \& Ervin-Tripp, 2006).

Tais concepções sugerem a importância do estudo da masculinidade e das normas de gênero para a compreensão das relações sociais entre homens e mulheres na atualidade. Nesse sentido, considerando a ausência de instrumentos na literatura brasileira acerca do tema que mensurem a concordância com normas de gênero, sugerindo diferentes concepções de masculinidade, o objetivo deste trabalho é adaptar a Escala de Concepções da Masculinidade para o contexto brasileiro. Para tanto, foram realizados dois estudos, apresentados a seguir. 


\section{ESTUDO 1}

Neste estudo pretendeu-se adaptar a Escala de Concepções da Masculinidade (ECM) para o contexto brasileiro, verificando seus parâmetros psicométricos.

\section{Método}

\section{Participantes}

Participaram do estudo 260 estudantes universitários, sendo $56,8 \%$ mulheres, com idade compreendida entre 17 e 49 anos $(M=22,2 ; D P=5,03)$. A maioria dos participantes se declarou solteira (90,3\%), estudante de universidades públicas $(69,1 \%)$ e de nível de religiosidade moderada $(M=3,46 ; D P=2,01)$.

\section{Instrumentos}

Para realização deste estudo os participantes responderam a um questionário formado pelos seguintes instrumentos:

Escala de Concep̧ỗes da Masculinidade (ECM). Desenvolvida por Oransky e Fisher (2009), originalmente em inglês, é composta por 26 itens que mensuram quatro fatores de masculinidade: esforço constante (ex.: Um homem sempre deveria parecer tão homem quanto outros que ele conhece); restrição emocional (ex.: Não é importante para homens escutar os problemas uns dos outros); heterossexismo (ex.: É difícil levar a sério um homem que usa esmalte de unha); e provocação social (ex.: Um homem não deveria se importar em ser zoado pelos amigos). Esses itens foram respondidos de acordo com uma escala tipo likert, variando entre 1 (Discordo) e 4 (Concordo).

Questões sociodemográficas. Para caracterizar os participantes do estudo foram incluídas questões como idade, sexo, religião, estado civil, tipo de universidade e, por último, uma pergunta sobre o nível de religiosidade (Você se considera uma pessoa religiosa?), que devia ser respondida de 1 (Nada religioso) a 7 (Muito religioso).

\section{Procedimentos}

Após aprovação do projeto de pesquisa pelo Comitê de Ética da Instituição, os questionários foram preparados para aplicação individual, mas em ambiente coletivo de sala de aula. Inicialmente, foram apresentadas informações sobre a pesquisa e o caráter anônimo da participação. A cada participante voluntário foi entregue o Termo de Consentimento Livre e Esclarecido, assegurando a confidencialidade de suas respostas. Foram necessários, em média, 20 minutos para preencher o instrumento.

\section{Análise de dados}

Estatísticas descritivas e as análises fatoriais exploratórias foram realizadas mediante o uso do software SPSS (versão 19). Para avaliação dos resultados das análises fatoriais exploratórias (AFE), foram utilizados os critérios propostos por Damásio (2012). A normalidade da distribuição dos itens na amostra foi testada, de forma a escolher a AFE mais apropriada a ser realizada, além de análises de alfa de Cronbach.

\section{Resultados e Discussão}

Inicialmente, utilizou-se a mediana empírica, tomando como base a pontuação total da escala, com a qual foram definidos os grupos critério inferior e superior, a fim de estabelecer o poder discriminativo dos itens. Para tanto, foi realizado um teste $t$ para cada item, comparando as médias dos dois grupos. Por não apresentar poder discriminativo satisfatório, o item 27 (Homens não zoam uns com os outros por maldade) foi retirado do instrumento.

Após verificar a adequabilidade da amostra à realização da análise fatorial $[K M O=0,86$; Teste de Esfericidade de Bartlett, $\chi^{2}$ (351) $\left.=2165,98, p<0,001\right]$, um teste de Kolmogorov-Smirnov (K-S) foi realizado para verificar se os itens da escala apresentam distribuição normal na amostra. Como a distribuição normal não foi encontrada (K-S $p<0,05)$, foi realizada uma análise dos eixos principais - PAF (Damásio, 2012), com rotação Varimax, solicitando 4 fatores, seguindo a sugestão de rotação e número de fatores dos autores do instrumento original (Oransky \& Fisher, 2009). Os resultados dessa análise indicaram que o quarto fator só incluiu dois itens, sugerindo que esta solução não pode ser considerada adequada. Uma análise paralela foi realizada, utilizando uma sintaxe do software SPSS, para definição do número de fatores a ser mantido, conforme sugerido por Damásio (2012). Esta análise sugeriu a existência de um único fator, com eigenvalue real igual a 6,47 , considerando $95 \%$ do intervalo de confiança.

Assim, uma nova PAF foi realizada, solicitando uma solução unifatorial e cargas fatoriais acima de $|0,40|$, de forma a garantir que apenas os melhores itens seriam mantidos nos fatores. O resultado de tal análise apresenta um fator com eigenvalue igual a 6,35, com $24,43 \%$ da variância explicada e a exclusão de nove itens por não alcançarem o valor mínimo (todos os cinco itens do fator provocação social, três itens do fator restrição emocional e um item do fator heterossexismo). No entanto, tal solução fatorial exclui integralmente 
uma dimensão considerada importante das concepções de masculinidade, que é a provocação social, não sendo considerada aceitável.

Uma terceira PAF foi realizada solicitando três fatores, conforme sugerido pela primeira PAF conduzida neste estudo, com rotação varimax (Oransky \& Fisher, 2009). Do total, sete itens foram excluídos por apresentarem cargas fatoriais abaixo do valor estipulado. Os 19 itens restantes apresentaram uma variância total explicada de $34,57 \%$. Apesar do quarto fator proposto teoricamente não ter sido observado, o conteúdo foi mantido e os itens referentes a essa dimensão dividiram-se entre os três fatores (ver Tabela 1). Como observado na Tabela 1, os índices de consistência interna dos três fatores são considerados adequados.

Esta análise indica que a ECM é uma escala que apresenta itens discriminativos, que se agrupam em fatores internamente consistentes para a mensuração das concepções de masculinidade dos participantes. A escala final deste estudo é uma versão reduzida, composta por 19 itens com as melhores saturações (cargas fatoriais acima de $|0,40|)$.

Os resultados descritos neste estudo diferem da estrutura proposta por Oransky e Fisher (2009). No processo de adaptação e análise dos dados, apenas três dimensões foram mantidas. Nesse sentido, torna-se importante demonstrar a adequação dessa nova versão, mediante a realização de um segundo estudo, utilizando para isso uma análise fatorial confirmatória.

\section{ESTUDO 2}

Este estudo teve como objetivo confirmar a estrutura fatorial da versão reduzida da Escala de Concepções da Masculinidade (ECM) no contexto brasileiro. Para tanto, um novo trabalho foi conduzido, tendo como objetivos secundários verificar a associação

Tabela 1. Estrutura fatorial da Escala de Concepções da Masculinidade (ECM)

\begin{tabular}{|c|c|c|c|}
\hline Itens & I & II & III \\
\hline 08. Aparentar ser gay faz com que um homem pareça menos homem & 0,71 & & \\
\hline 26. Homens de verdade nunca agem como uma menina & 0,70 & & \\
\hline 05. É embaraçoso ter muitos amigos gays & 0,69 & & \\
\hline 15. Uma boa maneira de parecer homem é evitar agir como um gay & 0,66 & & \\
\hline 18. Um homem deveria se sentir envergonhado de correr como uma garota & 0,61 & & \\
\hline 03. É difícil levar a sério um homem que usa esmalte de unha & 0,56 & & \\
\hline 22. Existe algo de errado se um homem quer fazer uma atividade feita por mulheres & 0,53 & & \\
\hline 10. Agir como homem deveria ser o objetivo mais importante para rapazes & 0,51 & & \\
\hline 17. Quando um homem sente medo, deveria manter isso pra si mesmo & & 0,66 & \\
\hline 16. Não importa o que aconteça, um homem deve aparentar ser forte para outros & & 0,62 & \\
\hline 11. Homens não deveriam falar sobre suas preocupações uns com os outros & & 0,48 & \\
\hline 21. É difícil respeitar um homem que demonstra seus sentimentos & & 0,48 & \\
\hline 06. Um homem nunca deveria recusar um desafio em público & & 0,41 & \\
\hline 14. Não é tarefa de um homem confortar um amigo chateado & & 0,40 & \\
\hline 09. Não tem nada de errado com um homem que zoa os seus amigos & & & 0,66 \\
\hline 19. É normal para os homens zoar seus amigos & & & 0,60 \\
\hline 23. Para ser aceito, os homens devem ser capazes de zoar outros & & & 0,51 \\
\hline 04. Um homem não deveria se importar em ser zoado pelos amigos & & & 0,45 \\
\hline 20. Ser zoado ajuda os homens a se tornarem durões & & & 0,43 \\
\hline Número de itens & 8 & 6 & 5 \\
\hline Eigenvalue & 4,17 & 2,78 & 2,04 \\
\hline \% de variância explicada & 16,03 & 10,68 & 7,86 \\
\hline Alfa de Cronbach & 0,86 & 0,72 & 0,69 \\
\hline
\end{tabular}


das dimensões finais com a idade, o sexo, a religiosidade e o nível de desejabilidade social dos participantes.

\section{Método}

\section{Participantes}

Participaram deste estudo 220 pessoas usuárias de internet, sendo 53,4\% mulheres, com idade compreendida entre 17 e 65 anos $(M=27 ; D P=8,35)$. A maioria dos participantes se declarou solteira $(51,6 \%)$, estudante de universidades públicas $(57,7 \%)$, com renda entre 1 e 5 salários mínimos $(50 \%)$, católica $(35 \%)$ e com um nível de religiosidade moderado $(M=3,68 ; D P=1,97)$.

\section{Instrumentos}

Os participantes responderam a um questionário formado pelos seguintes instrumentos:

Escala de Concepcôes da Masculinidade (ECM). Em sua versão reduzida, conforme resultado do Estudo 1, sendo composta por 19 itens que devem ser respondidos de acordo com uma escala tipo likert, variando entre 1 (Discordo) e 4 (Concordo). Os índices de consistência interna nessa amostra são relatados na seção de resultados.

Escala de Desejabilidade Social Marlowe-Crowne (EDSMC). Validada, em sua versão reduzida, no contexto brasileiro por Gouveia, Guerra, Sousa, Santos e Costa (2009), é composta por 20 itens que devem ser respondidos de acordo com o que o participante acredita ser verdadeiro (1) ou falso (0), no que diz respeito ao seu comportamento cotidiano. Esta versão apresenta um índice de consistência interna considerado adequado (Kuder-Richardson=0,76).

Questôes sociodemográficas. Para caracterizar os participantes do estudo foram incluídas questões como idade, sexo, religião, estado civil, tipo de universidade e, por último, uma pergunta sobre o nível de religiosidade (Você se considera uma pessoa religiosa?), que devia ser respondida de 1 (Nada religioso) a 7 (Muito religioso).

\section{Procedimentos}

Os questionários foram construídos eletronicamente para uma coleta via internet. $\mathrm{O}$ estudo foi divulgado por e-mail, entre contatos pessoais, e em redes sociais, sendo disponibilizado online por 3 meses. A página de apresentação do estudo continha as informações sobre a pesquisa e o caráter anônimo e voluntário da participação. Ao final dessa página, era solicitado o consentimento dos leitores para participação na pesquisa. Aqueles que concordavam seguiam para a primeira página do questionário, enquanto aqueles que discordavam eram levados a uma página de agradecimento pelo interesse na pesquisa. Em média, 20 minutos foram suficientes para concluir a participação.

\section{Análise de dados}

Estatísticas descritivas e correlações foram realizadas utilizando o software SPSS (versão 19). A análise fatorial confirmatória foi realizada mediante o uso do software $A M O S$, no qual se considerou a matriz de covariância com o estimador de máxima verossimilhança (ML). Foram utilizados os seguintes indicadores de ajuste do modelo aos dados: a razão qui-quadrado por graus de liberdade $\left(\chi^{2} / \mathrm{gl}\right)$, que deve apresentar resultados abaixo de 3; o goodness-of-fit index (GFI) e o comparative fit index (CFI), que devem apresentar resultados iguais ou acima de 0,90; e o root mean square error of approximation (RMSE $A$ ), que deve ser igual ou abaixo de 0,08 (Byrne, 2001).

\section{Resultados}

Com o objetivo de confirmar a estrutura fatorial da versão reduzida da ECM, três modelos foram elaborados: Modelo 1 - modelo com quatro fatores, baseado na proposta teórica original (Oransky \& Fisher, 2009); Modelo 2 - modelo de três fatores observado na AFE; e Modelo 3 - modelo unifatorial, proposto pela análise paralela. Após a testagem dos modelos, três itens foram excluídos por não apresentarem cargas fatoriais significativas (item 4, do fator provocação social; item 6 , do fator esforço constante; e item 14 , do fator restrição emocional). As análises foram realizadas novamente sem esses itens.

Os resultados dos índices de adequação de ajuste dos modelos aos dados, apresentados na Tabela 2, sugerem a inadequação do Modelo 3 (unifatorial) proposto pela análise paralela no Estudo 1. O Modelo 1, baseado na proposta teórica original, apresentou valores muito próximos daqueles considerados adequados em cada índice. Índices com valores apropriados foram observados no Modelo 2, baseado nos resultados do Estudo 1. No entanto, não foi observada diferença significativa entre os Modelos 1 (com quatro fatores) e 2 (com três fatores).

Em seguida, foram realizadas análises de consistência interna para os fatores dos dois modelos, de forma a identificar o que apresenta melhor consistência interna. Os índices observados para os fatores 
do Modelo 1 foram: heterossexismo $\alpha=0,87$; restrição emocional $\alpha=0,58$; provocação social $\alpha=0,62$; e esforço constante $\alpha=0,54$. os índices observados para os fatores do modelo 2 foram: heterossexismo $\alpha=0,87$; restrição emocional $\alpha=0,70$; provocação social $\alpha=0,65$. Com base nesses valores, o modelo de três fatores (ver Figura 1) foi escolhido para as análises subsequentes.

Com o modelo considerado adequado para utilização no contexto brasileiro, foram analisadas suas correlações com o nível de desejabilidade social dos participantes, assim como sua idade e nível de religiosidade. Os resultados dessas análises podem ser observados na Tabela 3.

As três dimensões apresentam-se positivamente correlacionadas entre si. No entanto, apenas provocação social apresentou correlações significativas (e negativas) com o nível de desejabilidade e a idade dos participantes. Ou seja, quanto maior a tendência a apresentar desejabilidade social e quanto maior a idade do participante, menor a tendência a concordar com a provocação social.

De forma a investigar diferenças na importância atribuída às dimensões de masculinidade e a relação destas com o sexo dos participantes, foi realizada uma análise mista de variância com design 3 (masculinidade: heterossexismo, restrição emocional, provocação social) x 2 (sexo: masculino, feminino). Os resultados dessa análise apresentaram um efeito principal da masculinidade, $f(2,432)=112,92, p<0,01, \eta^{2}$ parcial $=0,34$, com as dimensões heterossexismo e provocação social apresentando as maiores médias, semelhantes entre si, mas significativamente superiores à média de restrição emocional (ver Tabela 4).

Também foi observado um efeito de interação da masculinidade com o sexo dos participantes, $F(2$, $432)=3,42, p<0,05, \eta^{2}=0,03$. No geral, os homens apresentaram médias superiores de masculinidade em todas as dimensões, quando comparadas com as médias das mulheres. No entanto, os homens apresentaram

Tabela 2. Análises fatoriais confirmatórias da estrutura da Escala de Concepções da Masculinidade

\begin{tabular}{lcccccccc}
\hline Modelos & $\mathrm{gl}$ & $\chi^{2}$ & $\chi^{2} / \mathrm{gl}$ & GFI & CFI & RMSEA & D $\chi^{2}$ & Dgl \\
\hline Modelo 1 & 97 & $208,32^{* *}$ & 2,15 & 0,89 & 0,91 & 0,073 & - & - \\
Modelo 2 & 99 & $207,21^{* *}$ & 2,09 & 0,90 & 0,91 & 0,071 & 1,11 & 2 \\
Modelo 3 & 102 & $255,48^{* *}$ & 2,50 & 0,87 & 0,88 & 0,083 & $47,16^{* *}$ & 5 \\
\hline
\end{tabular}

Nota. ${ }^{* *} p<0,01$. Modelo 1 - original, com 4 fatores; Modelo 2 - 3 fatores; Modelo 3 - unifatorial.

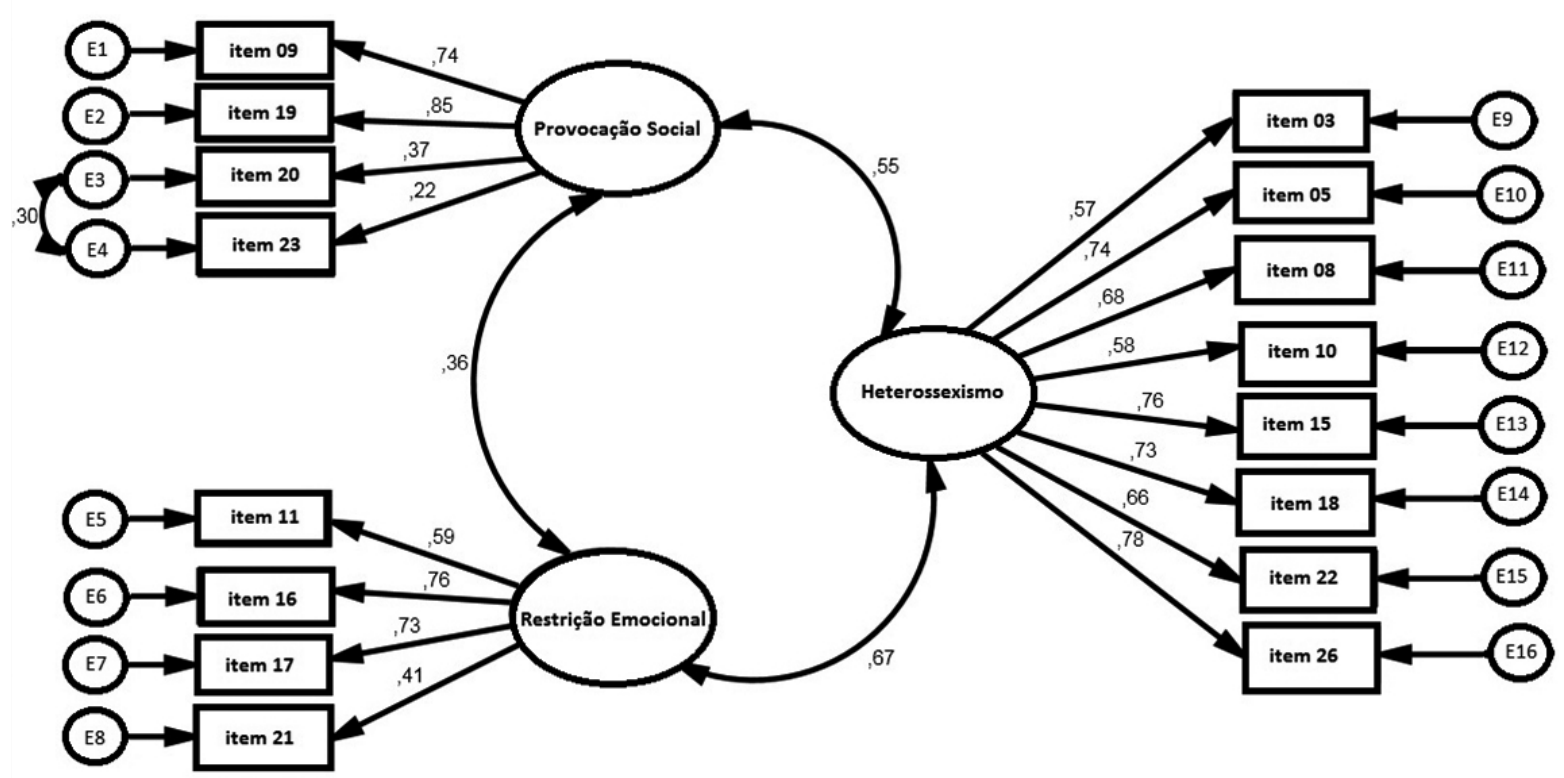

Figura 1. Estrutura fatorial da Escala de Concepções da Masculinidade 
Tabela 3. Correlação entre as dimensões de masculinidade, desejabilidade social, idade e religiosidade

\begin{tabular}{lccccc}
\hline Variáveis & HS & RE & PS & DS & Idade \\
\hline Heterossexismo & - & & & & \\
Restrição emocional & $0,55^{* *}$ & - & & & \\
Provocação social & $0,48^{* *}$ & $0,37^{* *}$ & - & & \\
Desejabilidade social & $-0,11$ & $-0,03$ & $-0,16^{*}$ & - & - \\
Idade & $-0,06$ & 0,08 & $-0,17^{*}$ & 0,04 & 0,05 \\
Religiosidade & 0,07 & 0,01 & $-0,04$ & $0,15^{*}$ & \\
\hline
\end{tabular}

Nota. $* p<0,05 ; * * p<0,01$. HS=Heterossexismo; RE=Restrição emocional; PS=Provocação social; DS=Desejabilidade social.

Tabela 4. Médias das dimensões de masculinidade de acordo com o sexo dos participantes

\begin{tabular}{lcccc}
\hline Sexo & Heterossexismo & Restrição emocional & Provocação social & Total \\
\hline Masculino & 2,30 & 1,60 & 2,16 & 2,02 \\
Feminino & 1,76 & 1,22 & 1,85 & 1,61 \\
Total & 2,01 & 1,40 & 2,00 & - \\
\hline
\end{tabular}

maior concordância com os itens de Heterossexismo, enquanto as mulheres apresentaram maior concordância com a provocação social.

\section{Discussão}

Considerando a importância do estudo da masculinidade e das normas de gênero para a compreensão das relações sociais entre homens e mulheres na atualidade e, ainda, considerando a ausência de instrumentos na literatura brasileira acerca do tema que mensurem a concordância com normas de gênero, sugerindo diferentes concepções de masculinidade, o objetivo deste trabalho foi adaptar a Escala de Concepções da Masculinidade para o contexto brasileiro.

A estrutura proposta pelos autores da escala original continha 26 itens divididos em quatro dimensões principais (heterossexismo, esforço constante, provocação social e restrição emocional). Contudo, tal estrutura não foi observada nas análises fatoriais exploratórias realizadas no Estudo 1, apontando para a necessidade de adaptação e reformulação da proposta. O Estudo 2 confirmou a estrutura observada no Estudo 1, indicando, para o contexto brasileiro, um modelo composto por 16 itens e três fatores.

Os itens que pertenciam à dimensão esforço constante, que enfatiza a necessidade de demonstrar força e confiança para demonstrar masculinidade, foram absorvidos pelos outros fatores. O item 10 ("Agir como homem deveria ser o objetivo mais importante para rapazes") associou-se ao fator heterossexismo. O conteúdo desse fator enfatiza o comportamento observável dos homens como essencial para a masculinidade. Para ser considerado 'homem', um rapaz não pode apresentar comportamentos considerados femininos. $\mathrm{O}$ item em questão refere-se a agir como homem, conteúdo este associado aos papéis de gênero expressos pela normatização da heterossexualidade (Scardua \& Souza Filho, 2006).

O item 16 ("Não importa o que aconteça, um homem deve aparentar ser forte para outros") associou-se ao fator restrição emocional. Em termos de conteúdo, esta dimensão enfatiza a necessidade de esconder as emoções e não aparentar vulnerabilidade. Da mesma forma que o anterior, este item apresenta associação direta com o conteúdo teórico do fator.

Finalmente, o item 20 ("Ser zoado ajuda os homens a se tornarem durões") associou-se ao fator provocação social, caracterizado pela ênfase em brincadeiras e piadas que fazem parte do papel de gênero masculino. Como pode ser percebido, cada item da dimensão esforço constante associou-se ao fator que apresentava o conteúdo mais próximo de seu significado original. Essa associação poderia indicar que, para esta amostra brasileira, cada uma das dimensões de masculinidade apresenta pelo menos uma indicação do 
esforço constante envolvido na manutenção do papel de gênero masculino: o esforço de evitar comportamentos inadequados, para manter o heterossexismo; o esforço de aparentar força todo o tempo, restringindo a expressão de suas emoções; e o esforço de criar piadas e aceitar ser o alvo de piadas dos amigos como parte do processo de construção desse papel social (Oransky \& Fisher, 2009).

Apesar da estrutura proposta pelos autores da escala original não ter sido encontrada, os resultados das análises fatoriais confirmatórias sugerem que, quando testada, a estrutura de quatro fatores não apresenta diferença significativa da estrutura de três fatores observada empiricamente. Não foram encontrados outros estudos acerca da estrutura da escala. Sendo assim, novos estudos são necessários para verificar a estabilidade das quatro dimensões propostas teoricamente. Nesse sentido, apesar de não seguir o modelo teórico, este modelo com três fatores é considerado mais apropriado para a amostra brasileira em questão por não excluir conteúdo teórico, por apresentar consistência interna e outros parâmetros psicométricos aceitáveis, além de indicadores de ajuste adequados que atendem aos valores sugeridos pela literatura (Byrne, 2001).

Portanto, este instrumento pode contribuir para uma melhor compreensão do fenômeno da masculinidade. Entretanto, como o estudo da validade de um instrumento é um processo contínuo de análise, novas pesquisas são sugeridas, especialmente no que diz respeito à sua estrutura multidimensional.

Em virtude de diferenças observadas no que diz respeito à estrutura do instrumento, é importante considerar possíveis limitações dos estudos que podem ter afetado tais resultados. As amostras não podem ser consideradas representativas da população brasileira como um todo: o Estudo 1 foi constituído exclusivamente por estudantes universitários e o Estudo 2, por usuários da internet. É necessário considerar que a constituição das amostras pode ter afetado diretamente a estruturação multidimensional dos itens. Nesse sentido, é necessário investigar participantes de amostras diferentes, de forma a testar se a estrutura fatorial observada será novamente confirmada. No entanto, apesar de tais diferenças e limitações, as três dimensões observadas nos estudos aqui relatados corroboram a concepção da masculinidade como um construto multidimensional (Mahalik e cols., 2003; Oransky \& Fisher, 2009).

No que diz respeito a estudos futuros, sugere-se investigar a associação das concepções da masculinidade com outros construtos, tais como a preocupação com a honra, os valores humanos, entre outros, abordando outras dimensões também pertencentes ao fenômeno da masculinidade e seu exercício na sociedade brasileira. Concebida enquanto um código comportamental que expressa a reputação de um indivíduo aos olhos de outras pessoas, a honra apresenta-se teórica e empiricamente associada aos papéis de gênero e, consequentemente, à masculinidade (Rodriguez Mosquera, Manstead \& Fischer, 2002).

Por fim, pesquisas futuras poderiam investigar o aumento atual de homens que buscam aperfeiçoar seu corpo para adequar-se aos padrões de beleza - o que sugere, a princípio, uma modificação na concepção de masculinidade. Um levantamento realizado por Wong, Steinfeldt, Speight e Hickman (2010) sobre o conteúdo da revista Psychology of Men \& Masculinity demonstrou a importância de se discutir a masculinidade e sua associação com a imagem corporal. Estariam a busca pelo corpo masculino perfeito e sua consequente auto-objetificação associadas de alguma forma à masculinidade e à conformidade às normas masculinas de gênero?

\section{Referências}

Bem, S. L. (1974). The measurement of psychological androgyny. Journal of Consulting and Clinical Psychology, 42 (2), 155 - 162.

Blazina, C., Pisecco, S., \& O’Neil, J. M. (2005). An adaptation of the Gender Role Conflict Scale for adolescents: psychometric issues and correlates with psychological distress. Psychology of Men \& Masculinity, 6, 39 - 45.

Bonomo, M., Barbosa, P. V., \& Trindade, Z. A. (2008). Homens: gênero e identidade em grupos tradicionais, metrossexuais e homossexuais no Brasil. Revista Electrónica de Psicología Política, 6(17), 1-22.

Byrne, B. M. (2001). Structural equation modeling with AMOS: basic concepts, applications and programming. Mahwah, NJ: Erlbaum.

Cochran, S. V. (2010). Emergence and development of the psychology of men and masculinity. Em J. C. Chrisler, \& D. R. McCreary. (Eds.), Handbook of gender research in Psychology (pp. 43-58). Nova Iorque: Springer.

Connell, R. W. (1997). La organización social de la masculinidad. Em Valdés, T. \& Olivarría, J. (Eds.). 
Masculinidad/es: poder y crisis (pp. 31-48). Santiago: Ediciones de las Mujeres.

Courtenay, W. H. (2000). Construction of masculinity and their influence on men's well-being: a theory of gender and health. Social Science \& Medicine, 50 (10),1385-1401.

Gouveia, V. V., Guerra, V. M., Sousa, D. M. F., Santos, W. S., \& Costa, J. M. (2009). Escala de Desejabilidade Social de Marlowe-Crowne: evidências de sua validade fatorial e consistência interna. Avaliaşão Psicológica, 8(1), 87-98.

Grossi, M. P. (2004). Masculinidades: uma revisão teórica. Antropologia em Primeira Mão, 75, 1-37.

Hernandez, J. A. E. (2009). Reavaliando o Bem Sex-Role Inventory. Estudos de Psicologia (Campinas), 26(1), 73-83.

Lampert, M. D. \& Ervin-Tripp, S. M. (2006). Risky laughter: teasing and self-directed joking among male and female friends. Journal of Pragmatics, 38, 51-72.

Louro, G. L. (2003). Gênero, sexualidade e educação - uma perspectiva pós-estruturalista. Petrópolis, RJ: Vozes.

Mahalik, J. R., Locke, B. D., Ludlow, L. H., Diemer, M. A., Scott, R. P. J., Gottfried, M., \& Freitas, G. (2003). Development of the conformity to Masculine Norms Inventory. Psychology of Men \& Masculinity, 4, 3 - 25.

Monteiro, M. (1997). A perspectiva do gênero nos estudos de masculinidade: uma análise da revista Ele Ela em 1969. Antropologia: Gênero e Masculinidade. Disponível em http://www.artnet.com.br/ marko/ artigo.html.

Murta, S. G., Del Prette, A., \& Del Prette, Z. A. P. (2010). Prevenção ao sexismo e ao heterossexismo entre adolescentes: contribuições do treinamento em habilidades de vida e habilidades sociais. Revista de Psicologia da Criança e do Adolescente, 2, 73-86.
Oransky, M. \& Fisher, C. (2009). The development and validation of the Meanings of Adolescent Masculinity Scale. Psychology of Men \& Masculinity, 10(1), 57-72.

Peter, A. S. (2010). The greatest quotations of all-time. Bloomington, Em Xlibris.

Rodríguez-Mosquera, P., Manstead, A., \& Fischer, A. (2002). The role of honour concerns in emotional reactions to offences. Cognition and Emotion, 16, 143-163.

Scardua, A. \& Souza Filho, E. A. (2006). O debate sobre a homossexualidade mediado por representações sociais: perspectivas homossexuais e heterossexuais. Psicologia: Reflexão e Crítica, 19(3), 482-490.

Tajfel, H. (1982). Social psychology of intergroup relations. Annual Review of Psychology, 33, 1-39.

Tsan, J. Y., Day, S. X., Schwartz, J. P., \& Kimbrel, N. A. (2011). Restrictive emotionality, BIS, BAS, and psychological help-seeking behavior. Psychology of Men \& Masculinity, 12(3), 260-274.

Vandello, J. A., Bosson, J. K., Cohen, D., Burnaford, R. M., \& Weaver, J. R. (2008). Precarious manhood. Journal of Personality and Social Psychology, 95(6), 1325-1339.

Wong, Y. J., Pituch, K. A., \& Rochlen, A. B. (2006). Men's restrictive emotionality: an investigation of associations with other emotion-related constructs, anxiety, and underlying dimensions. Psychology of Men \& Masculinity, 7(2), 113-126.

Wong, Y. J., Steinfeldt, J. A., Speight, Q. L., \& Hickman, S. J. (2010). Content analysis of psychology of men \& masculinity (2000-2008). Psychology of Men \& Masculinity, 11(3), 170-181.

Recebido em: 15/06/2013

Reformulado em: 12/11/2013

Aprovado em: 10/12/2013 
Sobre as autoras:

Valeschka Martins Guerra, doutora pela University of Kent em Canterbury, Inglaterra, é atualmente professora adjunta do Departamento de Psicologia Social e do Desenvolvimento e do Programa de Pós-Graduação em Psicologia da Universidade Federal do Espírito Santo. Sua experiência acadêmica centra-se na psicologia social, com ênfase em temas associados à sexualidade humana, valores, cultura, moralidade, psicologia positiva e instrumentação psicológica.

Arielle Sagrillo Scarpati é mestre em Psicologia pela Universidade Federal do Espírito Santo. Atualmente é doutoranda da University of Kent em Canterbury, Inglaterra. Sua experiência acadêmica centra-se na psicologia social e jurídica, com ênfase em temas associados à violência sexual contra a mulher, adoção, gênero e honra.

Camila Nogueira Bonfim Duarte é psicóloga pela Universidade Federal do Espírito Santo. Atualmente é mestranda da mesma universidade. Sua experiência acadêmica centra-se na psicologia social e da saúde, com ênfase em temas associados à violência contra a mulher, gênero, masculinidade, adoção e psicologia da gravidez.

Cleidiane Vitória da Silva é psicóloga pela Universidade Federal do Espírito Santo. Sua experiência acadêmica centra-se na psicologia social e da saúde, e psicologia do esporte.

Tammy Andrade Motta é psicóloga pela Univix (ES) e mestranda da Universidade Federal do Espírito Santo. Sua experiência acadêmica centra-se na psicologia social, com ênfase em temas associados ao gênero, honra, religiosidade e suicídio.

Contato com as autoras:

Valeschka Martins Guerra

Departamento de Psicologia Social e do Desenvolvimento

Cemuni VI, CCHN / UFES

Av. Fernando Ferrari, 514, Goiabeiras

Vitória - ES

29075-910

E-mail: valeschka.guerra@ufes.br 
